\title{
SYNTHETIC PHOTOMETRIC INDICES FOR GALACTIC
}

\section{GLOBULAR CLUSTERS}

\author{
M.L. MALAGNINI \\ Dipartimento di Astronomia, Universita' degli Studi di Trieste, Italy \\ L.E. PASINETTI FRACASSINI and S. COVINO \\ Dipartimento di Fisica, Universita' degli Studi di Milano, Italy \\ and \\ A. BUZZONI \\ Osservatorio Astronomico di Merate, Milano, Italy
}

\begin{abstract}
A grid of synthetic spectral energy distributions, representative of old stellar populations, has been used to derive colors in different photometric systems, and to compare the theoretical predictions with observational data for galactic globular clusters.
\end{abstract}

\section{Photometric indices}

Evolutionary synthesis of stellar populations provides a powerful tool for understanding the role of individual stellar components in the integrated radiation of stellar systems, such as clusters and galaxies, once the models have been proved to be consistent with the observations. In order to investigate this point, we undertook a systematic comparison between synthetic and observed photometric indices of galactic globular clusters. The synthetic indices were obtained from the integrated spectral energy distributions (SED's) for simple stellar populations (Buzzoni 1989), which have been computed by taking into account a set of relevant physical parameters, namely metal and Helium abundances $(\mathrm{Z}, \mathrm{Y})$; slope of the initial mass function (s); mass loss parameter $(\eta)$; and morphology of the horizontal branch (HB). A total number of 47 SED's have been considered; they were computed for ages $\tau=$ $10.0,12.5,15.0$, and $18.0 \mathrm{Gyrs}$, and the following values for the input parameters:

$-\quad \log \mathrm{Z}=-4.0(\mathrm{Y}=0.23),-3.0(\mathrm{Y}=0.23),-2.0(\mathrm{Y}=0.25)$;

$-\quad \mathrm{s}=1.35,2.35$

$-\quad \eta=0.30,0.50$.

Out of 47 models, 44 refer to synthetic clusters having, at $\tau=15.0$ Gyrs, an intermediate type $\mathrm{HB}$ morphology, as in M3, while the remaining three models have $\mathrm{HB}$ morphologies Blue (NGC 6752 like; $\log \mathrm{Z}=-4.0$ ) or Red (47 Tuc like; $\log \mathrm{Z}=$ $-3.0,-2.0)$, and fixed values $\mathrm{s}=2.35$ and $\eta=0.30$.

The photometric indices are those of Johnson UBVRIJK, Thuan and Gunn uvgr, and Washington Observatory $\mathrm{CMT}_{1} \mathrm{~T}_{2}$ systems. We ought to limit our analyses to photometric bands for which synthetic values could be computed from theoretical SED's, by taking into account their wavelength sampling.

From the observational point of view, a total number of 130 galactic globular clusters have been considered; the sample includes globular clusters with different morphologies of their HR diagrams. For each cluster, the following data have been collected, when available, the colors in the above-mentioned photometric systems, the $\mathrm{E}(\mathrm{B}-\mathrm{V})$ color excess, the integrated spectral type, the total visual magnitude, 
and the parameter $\mathrm{B} / \mathrm{B}+\mathrm{R}$. In order to make comparable the synthetic colors with the observed ones, we corrected the observational data for interstellar reddening.

First of all, the role of physical input parameters on the derived SED's has been considered, then synthetic colors have been computed, and their behavior in twocolors diagrams has been analyzed. Then comparisons have been made by using all available photometric data with the whole set of synthetic indices.

Here we present some results, in particular for the UBV system, referring to model predictions and to comparisons with observational data.

\section{Acknowledgements}

Partial support from MURST $40 \%-60 \%$, and CNR bilateral grants is acknowledged.

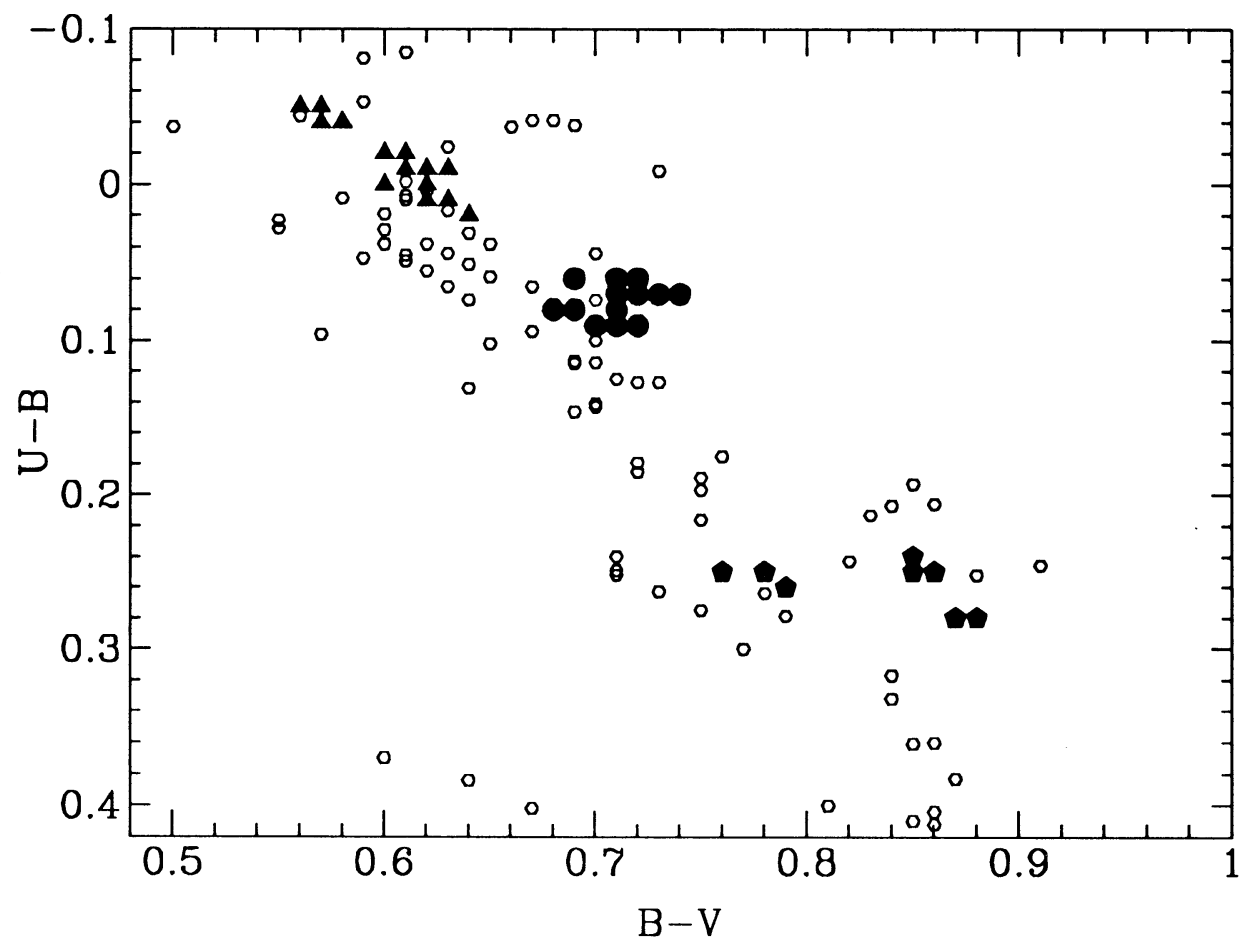

Fig. 1. Observed (open symbols) and computed (solid symbols) colors

\section{References}

Buzzoni, A., 1989, Astrophys. J. Suppl. Ser.,71, 817 